\title{
Development of Novel Patient-Derived Xenografts from Breast Cancer Brain Metastases
}

\author{
María J. Contreras-Zárate', D. Ryan Ormond ${ }^{2}$, Austin E. Gillen ${ }^{3}$, Colton Hanna', \\ Nicole L. Day', Natalie J. Serkova ${ }^{4}$, Britta M. Jacobsen', Susan M. Edgerton', \\ Ann D. Thor ${ }^{1}$, Virginia F. Borges ${ }^{5}$, Kevin O. Lillehei ${ }^{2}$, Michael W. Graner ${ }^{2}$, Peter Kabos ${ }^{5}$ \\ and Diana M. Cittelly ${ }^{1 *}$
}

\begin{abstract}
'Department of Pathology, University of Colorado Anschutz Medical Campus, Aurora, CO, United States, ${ }^{2}$ Department of Neurosurgery, University of Colorado Anschutz Medical Campus, Aurora, CO, United States, ${ }^{3}$ RNA Bioscience Initiative, University of Colorado Anschutz Medical Campus, Aurora, CO, United States, ${ }^{4}$ Department of Anesthesiology, University of Colorado Anschutz Medical Campus, Aurora, CO, United States, ${ }^{5}$ Department of Medicine, Division of Medical Oncology, University of Colorado Anschutz Medical Campus, Aurora, CO, United States
\end{abstract}

David D. Eisenstat, University of Alberta, Canada

Reviewed by:

Justin Lathia,

Cleveland Clinic Lerner College of

Medicine, United States

Ganesh Rao,

University of Texas MD Anderson

Cancer Center, United States

Carey Anders,

University of North Carolina at Chapel

Hill, United States

*Correspondence:

Diana M. Cittelly

diana.cittelly@ucdenver.edu

Specialty section:

This article was submitted

to Neuro-Oncology and

Neurosurgical Oncology,

a section of the journal

Frontiers in Oncology

Received: 17 July 2017

Accepted: 09 October 2017

Published: 02 November 2017

Citation:

Contreras-Zárate MJ, Ormond DR,

Gillen AE, Hanna C, Day NL,

Serkova NJ, Jacobsen BM, Edgerton $S M$, Thor $A D$, Borges VF,

Lillehei KO, Graner MW, Kabos P and Cittelly DM (2017) Development of

Novel Patient-Derived Xenografts from Breast Cancer Brain Metastases.

Front. Oncol. 7:252. doi: 10.3389/fonc.2017.00252
Brain metastases are an increasing burden among breast cancer patients, particularly for those with $\mathrm{HER}_{2}{ }^{+}$and triple negative (TN) subtypes. Mechanistic insight into the pathophysiology of brain metastases and preclinical validation of therapies has relied almost exclusively on intracardiac injection of brain-homing cells derived from highly aggressive TN MDA-MB-231 and HER2+ BT474 breast cancer cell lines. Yet, these well characterized models are far from representing the tumor heterogeneity observed clinically and, due to their fast progression in vivo, their suitability to validate therapies for established brain metastasis remains limited. The goal of this study was to develop and characterize novel human brain metastasis breast cancer patient-derived xenografts (BM-PDXs) to study the biology of brain metastasis and to serve as tools for testing novel therapeutic approaches. We obtained freshly resected brain metastases from consenting donors with breast cancer. Tissue was immediately implanted in the mammary fat pad of female immunocompromised mice and expanded as BM-PDXs. Brain metastases from 3/4 (75\%) TN, 1/1 (100\%) estrogen receptor positive (ER+), and 5/9 (55.5\%) HER2+ clinical subtypes were established as transplantable BM-PDXs. To facilitate tracking of metastatic dissemination using BM-PDXs, we labeled PDXdissociated cells with EGFP-luciferase followed by reimplantation in mice, and generated a BM-derived cell line (F2-7). Immunohistologic analyses demonstrated that parental and labeled BM-PDXs retained expression of critical clinical markers such as ER, progesterone receptor, epidermal growth factor receptor, HER2, and the basal cell marker cytokeratin 5. Similarly, RNA sequencing analysis showed clustering of parental, labeled BM-PDXs and their corresponding cell line derivative. Intracardiac injection of dissociated cells from BM-E22-1, resulted in magnetic resonance imaging-detectable macrometastases in 4/8 (50\%) and micrometastases (8/8) (100\%) mice, suggesting that BM-PDXs remain capable of colonizing the brain at high frequencies. Brain metastases developed 8-12 weeks after ic injection, located to the brain parenchyma, grew around blood vessels, and elicited astroglia activation characteristic of breast 
cancer brain metastasis. These novel BM-PDXs represent heterogeneous and clinically relevant models to study mechanisms of brain metastatic colonization, with the added benefit of a slower progression rate that makes them suitable for preclinical testing of drugs in therapeutic settings.

Keywords: patient-derived xenograft, brain metastases models, breast cancer, brain colonization, triple negative, HER2 ${ }^{+}$

\section{INTRODUCTION}

Brain metastases are the most common form of brain cancer, exceeding the number of primary brain tumors by at least four times, and occurring in about $25 \%$ of all patients with cancer (1). Breast cancer is the second most common primary tumor responsible for brain metastasis $(2,3)$, especially from women with $\mathrm{HER}^{+}$and triple negative [TN, estrogen receptor negative $\left(\mathrm{ER}^{-}\right)$, progesterone receptor negative $\left(\mathrm{PR}^{-}\right)$, and HER2 ${ }^{-}$] tumors (4-6). Brain metastases remain incurable and more than $80 \%$ of patients will die within a year of their brain-metastasis diagnosis $(7,8)$. Treating brain metastases has been particularly challenging due to unique anatomical and functional features in the brain. Therapies used to treat systemic metastases [e.g., trastuzumab for the treatment of breast tumors overexpressing HER $2^{+}$, or chemotherapies used to treat triple negative breast cancers (TNBCs)] have limited ability to cross the blood-brain barrier (BBB) at effective doses, and often fail to decrease brain metastatic burden $(8,9)$. Thus, there is an urgent need for improved therapeutic approaches for breast cancer brain metastases.

A critical limitation to achieve better therapeutic strategies for brain metastasis has been the narrow set of experimental models to study brain metastasis pathophysiology. Development of symptomatic brain metastasis requires cancer cells to disseminate from the primary tumor, intravasate into blood vessels, survive in circulation, extravasate through the BBB, survive the neuroinflammatory response in the brain, and outgrow into large metastasis (10-12). Studying this complex process requires in vivo animal models that mimic early and late stages of brain metastatic colonization, produce brain metastases at high frequencies, and demonstrate moderate tumor progression necessary for the preclinical screening of drugs that could be used in preventive and therapeutic settings $(13,14)$. Until recently, brain metastasis studies relied primarily on intracardiac (ic) injection of brain-homing cells derived from murine 4T1 (4T1BR5), human TN MDA-MB-231 (231Br, 231/LM2-4) (15-17), and HER2 ${ }^{+}$BT474 (BT474BR) cell lines (18). These models were developed by performing successive rounds of ic injection of breast cancer cell lines, which were then reisolated, cultured in vitro and then reinjected into nude mice (19). Although these brain metastatic cell lines are well characterized and produce brain metastases at high frequencies $(20,21)$, the rapid progression of metastatic burden in these models limits their usability for therapeutic testing of drugs. More importantly, these models do not fully represent the heterogeneity observed in breast tumors and their metastasis, which have emerged as critical factor in defining populations of patients that are likely to respond to a particular therapy.

During the past several years, researchers have developed transplantable models to grow primary breast tumors in the mammary fat pad of NOD/SCID/ILIIrg ${ }^{-/-}$(NSG) mice, with the long-term goal of personalizing medicine (22-24). These PDXs retain intratumoral heterogeneity and have become a clinically relevant alternative to cell lines $(23,25,26)$. Here, we report the development and characterization of eight novel human breast cancer patient-derived xenografts (BM-PDXs) from $\mathrm{ER}^{+}$, HER $^{+}$, and TN subtypes and a matching TN cell line, which retain tumor heterogeneity and brain metastatic potential. We demonstrate that ic injection of cells dissociated from BM-PDXs produce brain metastases at high frequencies, with metastases that elicit astroglia activation and growth around vessels in a similar fashion to breast cancer brain metastasis. These novel BM-PDXs represent heterogeneous and clinically relevant models to study mechanisms of brain metastatic colonization, with the added benefit of a slower progression rate that makes them suitable for preclinical testing of drugs in therapeutic settings.

\section{MATERIALS AND METHODS}

\section{Brain Metastases Transplantation and Establishment of Patient-Derived Xenografts}

De-identified brain metastases and their clinical-pathological information (age, ER, PR, and HER2 status at the time of metastases resection, prior therapies, and survival) were obtained from consenting breast cancer patients undergoing neurosurgery. These samples were collected under approved IRB protocols at University of Colorado Anschutz Medical Campus. All animal studies were performed under approved University of Colorado Institutional Animal Care and Use Committee (IACUC) protocols.

Freshly removed brain metastasis samples were placed on sterile ice-cold DMEM and transported to the laboratory for transplantation into mice. Specimens that could not be immediately implanted were maintained at $4^{\circ} \mathrm{C}$ for no longer than $8 \mathrm{~h}$. Female NSG mice, 6-8 weeks old were purchased from Jackson laboratories or bred at the UC Denver Center for Comparative Medicine breeding facility. Brain metastases were partitioned into $5-10 \mathrm{~mm}^{3}$ pieces, dipped into cultrex, and implanted in the fourth mammary fat pad of anesthesized mice using a 10-gage trochar. In one case, brain metastatic cells were collected from cerebrospinal 
fluid from a patient diagnosed with meningeal carcinomatosis. Here, cancer cells were collected by centrifugation and divided into two aliquots. One mouse was injected ic with cancer cells suspended in $100 \mu \mathrm{l}$ PBS, another recipient was injected into the mammary fat pad with cancer cells resuspended in $50 \mu \mathrm{l}$ cultrex. All mice were implanted with a silastic pellet providing slow release of $17 \beta$-estradiol (E2), as prior experience showed that E2 increases tumor uptake of breast cancer PDXs irrespective of tumor subtype. Tumors were palpated weekly to assess tumor take for up to 8 months postimplantation. Once palpable, tumor size was assessed weekly using caliper and volume estimated as length $\times$ width $^{2} / 2$. When tumors reached $\sim 1.5 \mathrm{~cm}$ in any direction, mice were euthanized and tumors removed. Tumors were divided into several $10 \mathrm{~mm}^{3}$ pieces and reimplanted in the mammary fat pad of NSG mice, cryopreserved in 10\% DMSO/90\% FBS in liquid nitrogen, stored in trizol RNA extraction, and fixed in $10 \%$ formalin for paraffin embedding. BM-PDXs were considered established if they grew over two generations.

\section{Labeling of BM-PDXs}

A subset of BM-PDXs were labeled with lentiviral particles expressing EGFP-luciferase as we described previously (27). Briefly, $>1 \mathrm{~cm}^{3}$ tumors were resected from euthanized mice and digested in Accumax (Stemcell Tech) for $3 \mathrm{~h}$ at $30^{\circ} \mathrm{C}$. Human cancer cells were separated from mouse stromal cells using a lineage cell depletion kit (MACS) and isolated breast cancer cells were plated in six-well ultralow attachment plates in DMEM-F12 media. Tumor cells were transduced with $30 \mathrm{MOI}$ of lentiviral pHAGE-EF1aL-luciferase-UBC-GFP-W and GFP expression monitored for up to $48 \mathrm{~h}$. Labeled tumor cells were then collected, washed, resuspended in $100 \mu \mathrm{l}$ Cultrex basement membrane extract and injected in the mammary fat pad of NSG mice. Efficiency of transduction was assessed using luciferase activity imaging (IVIS) or GFP expression when tumors reached $>1 \mathrm{~cm}^{3}$. Labeled BM-PDX were cryopreserved, fixed for immunohistological analysis, stored in trizol for RNA extraction, or transplanted into a new recipient.

\section{BM-F2-7 Cell Line Derivation and Culture}

A cell line (F2-7) was derived from triple-negative BM-PDX. For this, tumor cells were dissociated from BM-PDX F2-7 using Accumax, and dissociated cells were plated in ultralow attachment six-well plates in DMEM-F12 supplemented with $10 \%$ of FBS, $1 \mu \mathrm{g} / \mathrm{ml}$ hydrocortisone, $100 \mathrm{ng} / \mathrm{ml}$ of cholera toxin, and $1 \mathrm{nM}$ of insulin. After 4 weeks of growth in suspension, human cells free of fibroblasts were plated in collagen-I coated dishes, and purity validated by immunohistochemistry. F2-7 cells were labeled with GFP-luciferase as described for BM-PDXs. ShortTandem Repeat analysis was performed in the established cell line and deposited at University of Colorado Tissue Culture Core facility for validation and future reference.

\section{RNA Sequencing of BM-PDX and F2-7 Cell Line}

High-throughput RNA sequencing from a cell line derivative (F2-7) and a selected set of BM-PDXs before and after labeling with EGFP-luciferase was performed. RNA was isolated from tumor samples using trizol followed by RNA cleanup using RNEeasy MinElute Cleanup kit (Qiagen), and RNA concentration was measured in a Nanodrop 2000 (Thermo Scientific). The Genomics and Microarray core facility at the University of Colorado AMC performed RNA quality control using an Agilent 2100 Bioanalyzer, and prepared RNA-seq libraries using the Illumina TruSeq Stranded mRNA LT Sample Prep Kit. The resulting libraries were sequenced on an Illumina HiSeq 2500 system $(1 \times 125 \mathrm{bp})$. After demultiplexing, the resulting reads were trimmed with cutadapt to remove $3^{\prime}$ adaptor sequences and low quality $3^{\prime}$ bases $(\mathrm{Q}<10)$. The trimmed reads were then aligned to both the human (hg19/GRCh37) and mouse (mm10) genomes using Tophat2 (28). Reads were then assigned to either the human or mouse genome using disambiguate (29) and ambiguous reads were discarded. Unambiguous reads were assigned to features using Rsubread (30) and normalized read counts were produced using the rlog function in DESeq2 (31). The GEO Accession number for this data is GSE104020.

\section{Experimental Brain Metastasis Using BM-PDXs}

Two tumors from E22-1 BM-PDXs grown in the mammary fat pad were excised at necropsy, cut into $2 \mathrm{~mm}^{2}$ pieces and dissociated using Accumax for $3 \mathrm{~h}$. The digestion was stopped using DMEM/F12 10\% FBS, and single cells isolated by filtering through 100 and $70 \mu \mathrm{m}$ mesh filters. Viable cells were counted using trypan blue exclusion and 250,000 cells resuspended in $100 \mu \mathrm{l}$ PBS were injected in the left cardiac ventricle of recipient female NSG mice $(n=8)$. Brain metastatic burden was assessed using T1/T2 contrast magnetic resonance imaging (MRI) 8 weeks after ic injection, and mice were euthanized under $\mathrm{CO}_{2}$ asphyxiation at 8 or 12 weeks after ic injection as mice developed signs of CNS metastatic burden. In all cases, brains were removed at necropsy and brain hemispheres embedded in OCT and stored at $-80^{\circ} \mathrm{C}$ until sectioning. Micrometastases were visualized with $\mathrm{H} \& \mathrm{E}$ and/or pan cytokeratin (PanCK) staining in six serial sections (10 $\mu \mathrm{m}$ thick), one every $300 \mu \mathrm{m}$ in a sagittal plane through the right hemisphere of the brain.

\section{Magnetic Resonance Imaging}

To non-invasively detect and quantify brain metastatic colonization, brain MR scans were acquired using a Bruker $4.7 \mathrm{~T}$ PharmaScan and a bird-cage radio frequency $36 \mathrm{~mm}$ coil (Bruker Medical, MA, USA). Animals were injected via tail vain with $0.4 \mathrm{mmol} / \mathrm{kg}$ gadolinium contrast Multihance (gadobenate dimeglumine, Bracco Diagnostic) and anesthetized with 2-2.5\% isoflurane. High-resolution rapid acquisition with relaxation enhancement (RARE) T2-weighted images with fat suppression were obtained $(\mathrm{TR} / \mathrm{TE}=4,000 / 80 \mathrm{~ms})$ followed by a multislice multiecho (MSME) T1-weighted sequence (TR/TE $=700 / 11 \mathrm{~ms})$. All images were obtained in the axial plane, with the field of view of $3 \mathrm{~cm}$, slice thickness $1 \mathrm{~mm}$, number of slices 16 , matrix size $256 \times 256$. In-plane resolution was $90 \mu \mathrm{m}$. T1-weighted MSME images were acquired as well to confirm metastatic lozation. All 

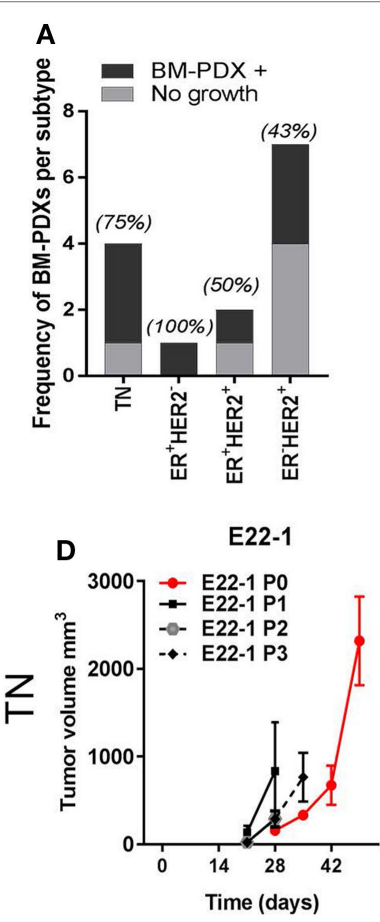

E

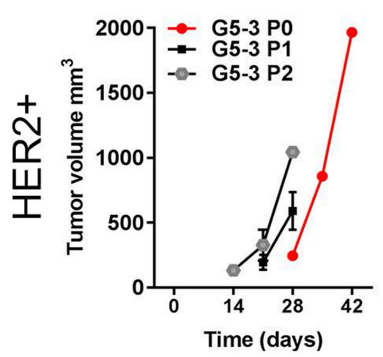

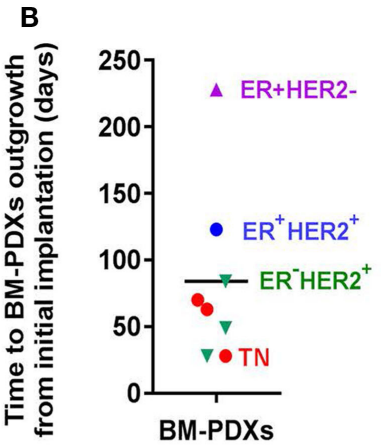

F2-7

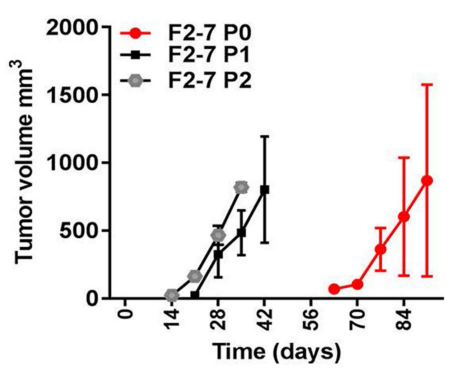

G6-9

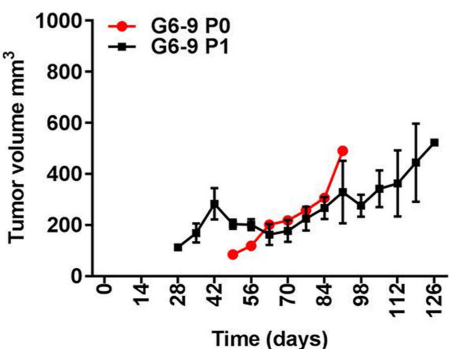

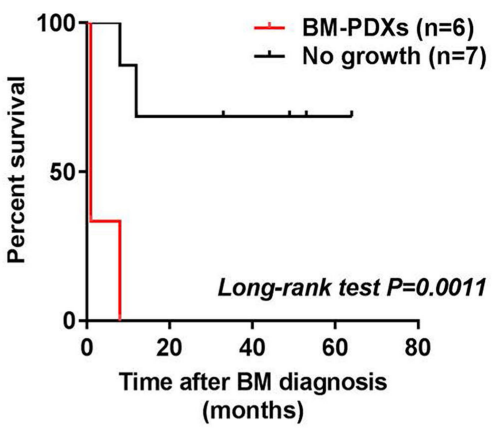

CSF-1

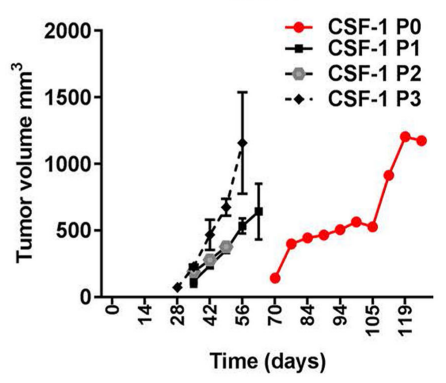

BM9-14

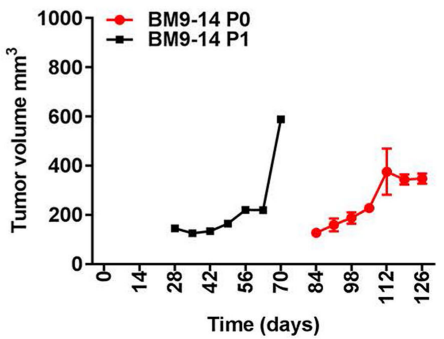

FIGURE 1 | Establishment of brain-metastases-patient-derived xenografts (BM-PDXs) from breast cancer. PDXs were established in NOD/SCID/ILIIrg ${ }^{-/-}$mice, and subsequently propagated via direct transplantation of solid tumor pieces into new recipient mice. Tumors were grown under continuous estrogen supplementation. (A) BM-PDXs established as a function of breast cancer subtypes. Graph depicts the number (bars) and percentage of BM-PDXs established (BM-PDXs ${ }^{+}$) per tumor subtype compared to tumors that did not grow after 8 months of implantation. (B) Time in days from initial implantation to outgrowth as measurable tumors $\left(\sim 62.5 \mathrm{~mm}^{3}\right)$ for all PDXs, colors indicate breast cancer subtypes. (C) Survival (months) after brain metastases diagnosis of patients with breast cancer whose surgical samples had in vivo tumorigenic potential $(n=6)$, or not $(n=7)$. Log-rank (Mantel-Cox) test $P$ value is shown. (D,E) Tumor growth after implantation (P0, red lines), and subsequent passaging (P1, P2, P3, black/gray lines) in (D) TN and (E) ER-HER2+ BM-PDXs. For P0, 1-2 tumors were implanted in a single recipient. For P1-P3, data shows average tumor volume from two tumors in 1-2 recipient mice.

images were acquired and analyzed (T2-MRI for lesion numbers and diameters) using Bruker ParaVision (v4.3) software.

\section{Immunohistochemistry in PDXs and Experimental Brain Metastasis}

Tumors were removed from animals and fixed in 10\% buffered formalin. Tissue was processed, paraffin embedded, and cut into $5-\mu \mathrm{m}$ sections. After high-temperature antigen retrieval in citrate buffer, sections were stained with rabbit anti-epidermal growth factor receptor (anti-EGFR, Cell Signaling), rabbit monoclonal anti-C-erB-2 (SP3, Neomarkers), mouse monoclonal antibody anti-cytokeratin 5 (anti-CK5, Vector), rabbit polyclonal anti-PR (DAKO, Carpinteria, CA, USA), and rabbit polyclonal anti-ER $\alpha$ (SP1, Thermofisher). Sections were counterstained with hematoxylin and mounted. Representative photographs were taken under a light microscope at $\times 20$ magnification.

Dual immunofluorescence of brain metastasis was performed $10-\mu \mathrm{m}$ sections from frozen unfixed-OCT embedded brains. Sections were fixed in acetone and stained with a mouse monoclonal antibody specific for human cytokeratins (Pan-CK, MNF116, Dakocytomation, Glostrup Denmark); in combination with rat anti-GFAP (Invitrogen, CA, USA); rabbit anti-collagen IV (Millipore). Secondary antibodies were anti-mouse Alexafluor-488 or anti-rabbit Alexafluor-565 or 
TABLE 1 | Clinical-pathological characteristics of BM-PDXs.

\begin{tabular}{|c|c|c|c|c|c|c|}
\hline \multicolumn{7}{|c|}{ Clinical features of BM-PDXs } \\
\hline \multirow[b]{2}{*}{ PDX } & \multirow[b]{2}{*}{$\begin{array}{l}\text { Primary } \\
\text { tumor Dx }\end{array}$} & \multirow[b]{2}{*}{ BM-Dx } & \multicolumn{4}{|c|}{ At brain metastasis diagnosis } \\
\hline & & & Age & ER & PR & HER2 \\
\hline E22-1 & $\mathrm{TN}$ & $\mathrm{TN}$ & 58 & 0 & 0 & 0 \\
\hline $\mathrm{F} 2-7$ & $\mathrm{TN}$ & $\mathrm{TN}$ & 38 & 0 & 0 & 0 \\
\hline BM14-9 & ER-HER2 ${ }^{+}$ & $\mathrm{ER}^{-} \mathrm{HER}^{+}{ }^{+}$ & 43 & 0 & 0 & $95 \%(3+)$ \\
\hline G5-3 & $\mathrm{TN}^{\mathrm{a}}$ & $\mathrm{ER}^{-\mathrm{HER}} 2^{+}$ & 63 & 0 & $5 \%(1+)$ & $30 \%(2+), \mathrm{FISH}^{+}$ \\
\hline G6-9 & $\mathrm{TN}^{\mathrm{a}}$ & $\mathrm{ER}^{-} \mathrm{HER} 2^{+}$ & 53 & 0 & 0 & $40 \%(2+)$ \\
\hline G7-1 & $\mathrm{ER}^{+} \mathrm{HER} 2^{+}$ & $\mathrm{ER}^{+} \mathrm{HER} 2^{+}$ & 59 & $65 \%(2+)$ & $70 \%(2+)$ & $45 \%(2+), \mathrm{FISH}^{+}$ \\
\hline G13-1 & $\mathrm{ER}^{+} \mathrm{HER} 2^{-}$ & ER+HER2- & 63 & $70 \%(3+)$ & 0 & 0 \\
\hline CSF $-1^{b}$ & $\mathrm{TN}$ & $\mathrm{TN}$ & ND & ND & ND & ND \\
\hline
\end{tabular}

Numbers represent $\%$ of positive cells followed by intensity score (in brackets). HER2+ is defined as $>10 \%$ and $\geq(2+)$. ER $R^{+}$is defined as $>1 \%$.

aPrimary tumors with history of TNBC that had converted to $\mathrm{HER} 2^{+}$at brain metastasis. ${ }^{b}$ Sample obtained from cerebrospinal fluid, no pathology report at metastatic site. All patients had been treated with taxanes by the time of brain metastases.

anti-Rat-Alexafluor-594 (all from Invitrogen/Thermofisher, CA, USA). Nuclei were stained with $1 \mu \mathrm{g} / \mathrm{ml} \mathrm{4',6-diamidino-2-phe-}$ nylindole (DAPI) in methanol for $10 \mathrm{~min}$ at room temperature. Photographs were taken under $\times 20$ magnification for the same field using the UV, FITC, and TRITC filters.

\section{RESULTS}

\section{Establishment of BM-PDXs in Relation to Breast Cancer Subtypes}

We implanted a total of 14 brain metastases specimens from TN $(n=4), \mathrm{ER}^{+} \mathrm{HER}^{-}(n=1), \mathrm{ER}^{+} \mathrm{HER}^{+}(n=2)$, and ER ${ }^{-} \mathrm{HER} 2^{+}$ $(n=7)$ breast cancer subtypes. From these, 8 (57.2\%) successfully established as BM-PDXs, defined as those tumors that grew as xenografts in NSG mice at least in one consecutive passage and maintained expression of clinical markers from original patient sample. The frequency of BM-PDXs uptake varied among subtypes with a non-significant trend toward highest take rate in TNBC (3/4 BM-PDXs, 75\%) and lower take rate in ER-HER2 ${ }^{+}(3 / 7$, 43\%) (Figure 1A). The overall clinical-pathological characteristics of the brain metastases successfully established as BM-PDXs are presented in Table 1. Among HER2 ${ }^{+} \mathrm{BM}-\mathrm{PDX}$, two specimens had prior history of TNBC but their brain metastasis were diagnosed as HER2 ${ }^{+}$; these were classified as HER2+BM-PDXs. Time to xenograft tumor formation for each BM-PDX at initial implantation ranked between 28 and 223 days, with an average of 84 days (Figure 1B). Similar to prior reports, in vivo tumorigenic potential of explanted brain metastases (tumors that grew as BM-PDXs) was correlated with decreased survival of their donor patients $(P=0.0011)$ (Figure 1C). Tumor progression in initial PDX (P0) and subsequent in vivo passaging (P1 to P3) for a TN and HER2 ${ }^{+} \mathrm{BM}-\mathrm{PDX}$ s is shown in Figures 1D,E. In one case, an ER-HER2 ${ }^{+}$brain metastases was implanted but an inguinal tumor developed suddenly after 70 days, away from the implantation site. This tumor lacked HER2, EGFR, or CK5 and upon transplantation grew into a large mass within 2 weeks (data not shown). As this suggested either loss of human epithelial markers or most likely, outgrowth of a murine tumor, we did not consider this a successful PDX and excluded it from further analysis.

\section{Preservation of Clinical Markers in BM-PDXs Over Multiple Passaging, Cell Dissociation, and Viral-Mediated Transduction}

To determine whether outgrowth of BM-PDXs in the mammary fat pad retained key clinical features of brain metastases donors, we stained sections of BM-PDXs (P0-P1) for ER, PR, and HER2, and-when available-we compared them to clinical specimens at the time of implantation. TNBC brain metastases lack these markers but frequently express EGFR and CK5 (32). Thus, we added these to our validation panel. As show in Figure 2A, TN BM-PDXs expressed EGFR and CK5. For these samples there was no matching donor sample to compare, but lacked ER, PR, and HER2 as expected from TN tumors. ER-HER2+BM-PDXs retained HER2, EGFR, and CK5 (Figure 2B), and ER+BM-PDXs retained ER, HER2, EGFR, and CK5 expression similar to the donor sample (Figure 2C). Surprisingly the two ER ${ }^{+} B M-P D X s$ (G7-1, G13-1), showed increased PR expression as compared to the donor samples (Figure 2C), suggesting that ER is functional in these BM-PDXs and that E2-supplementation in mice upregulates $\mathrm{PR}$ in $\mathrm{ER}^{+} \mathrm{BM}-\mathrm{PDX}$.

To assess whether GFP and luciferase labeling would allow in vivo imaging of these BM-PDXs, we dissociated cells from $>1 \mathrm{~cm}^{3}$ xenografts BM-PDXs F2-7, CSF-1, and G5-3 and transduced them with high titer viral particles of a GFP-luciferase vector as described (27). Labeled cells were regrown in the mammary fat pad of NSG mice and tumor labeling was assessed by measuring luciferase activity (IVIS) and GFP expression (Figure 3A). Spontaneous metastases to surrounding areas were detected in some mice during tumor excision (Figure 3B), but without spontaneous metastases to brain or other organs. Since tumor cell dissociation and cell transduction with lentiviral vector might results in selection of subclones of the original tumor, we assessed whether labeled PDXs recapitulated the heterogeneity observed in parental PDXs. IHC staining showed that labeled BM-PDXs retained expression of EGFR, CK5, and HER2 (Figure 3C), suggesting that dissociated/labeled cells are capable of reconstituting tumor heterogeneity of BM-PDXs.

As tumor-dissociated cells survived short-term in vitro culture during labeling, we sought to determine whether dissociated brain-metastatic cells could be cultured as cell lines. We cultured dissociated cells from F2-7, E22, and G5-3 BM-PDXs in plates coated with collagen-I or ultralow attachment plates. In either condition, only cells from F2-7 BM-PDXs survived in vitro culture and remained proliferative after multiple passages of repeated freezing and thawing (Figure 4A). This F2-7 cell line-derivative retained expression of EGFR as its BM-PDXs counterpart (Figure 4B), and retained tumor initiating capability in vitro (measured as ability to form colonies in the absence of extracellular matrix in mammosphere assays, not shown). To further assess whether BM-PDXs labeling or cell line derivation 
maintained features of parental BM-PDXs, we performed RNA sequencing followed by hierarchical gene clustering analysis of BM-PDX before and after GFP-luciferase labeling, and F2-7 BM-PDX and its cell line-derivative (Figure 5). Key genes (EGFR, KRT5, NTRK2) were expressed at similar levels in parental PDXs (i.e., E22-1 PDX-P0) compared to its labeled counterpart (E22-1 PDX-P0-I1), and in the F2-7 cell line (F2-7 CL) compared to its BM-PDXs parental (F2-7 P5). Taken together, these data suggests maintenance of clinical markers through passaging and manipulation of BM-PDXs.

\section{BM-PDXs Retain Their Ability to Colonize the Brain at High Frequencies}

Breast cancer PDXs grown in the mammary fat pad rarely metastasize to distant organs, but dissociated cells can colonize lung, bones and brain after ic injection (27). To assess whether BM-PDXs retain their ability to colonize the brain, we induced experimental brain metastasis using dissociated cells from TN E22-1 BM-PDX. For this, 250,000 dissociated cells were injected in the left ventricle of 8 -week-old female NSG mice $(n=8)$ supplemented with estradiol, and metastases were allowed to grow
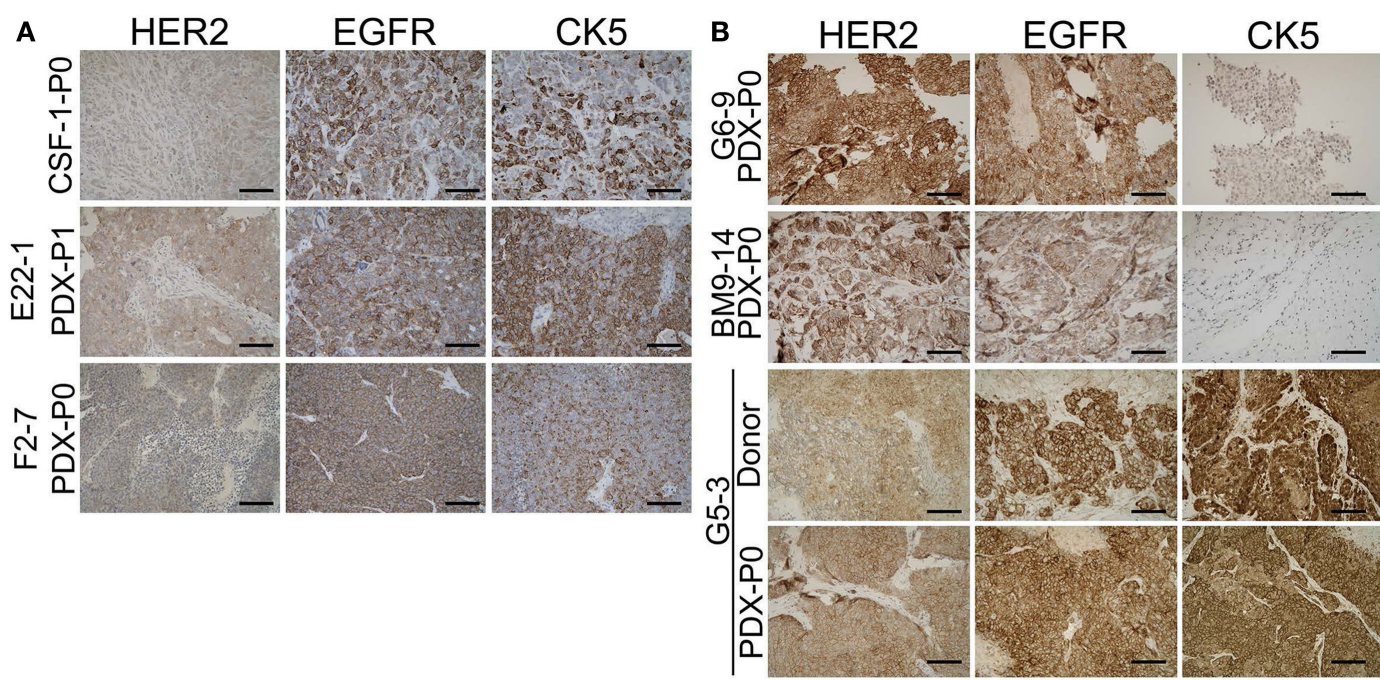

C
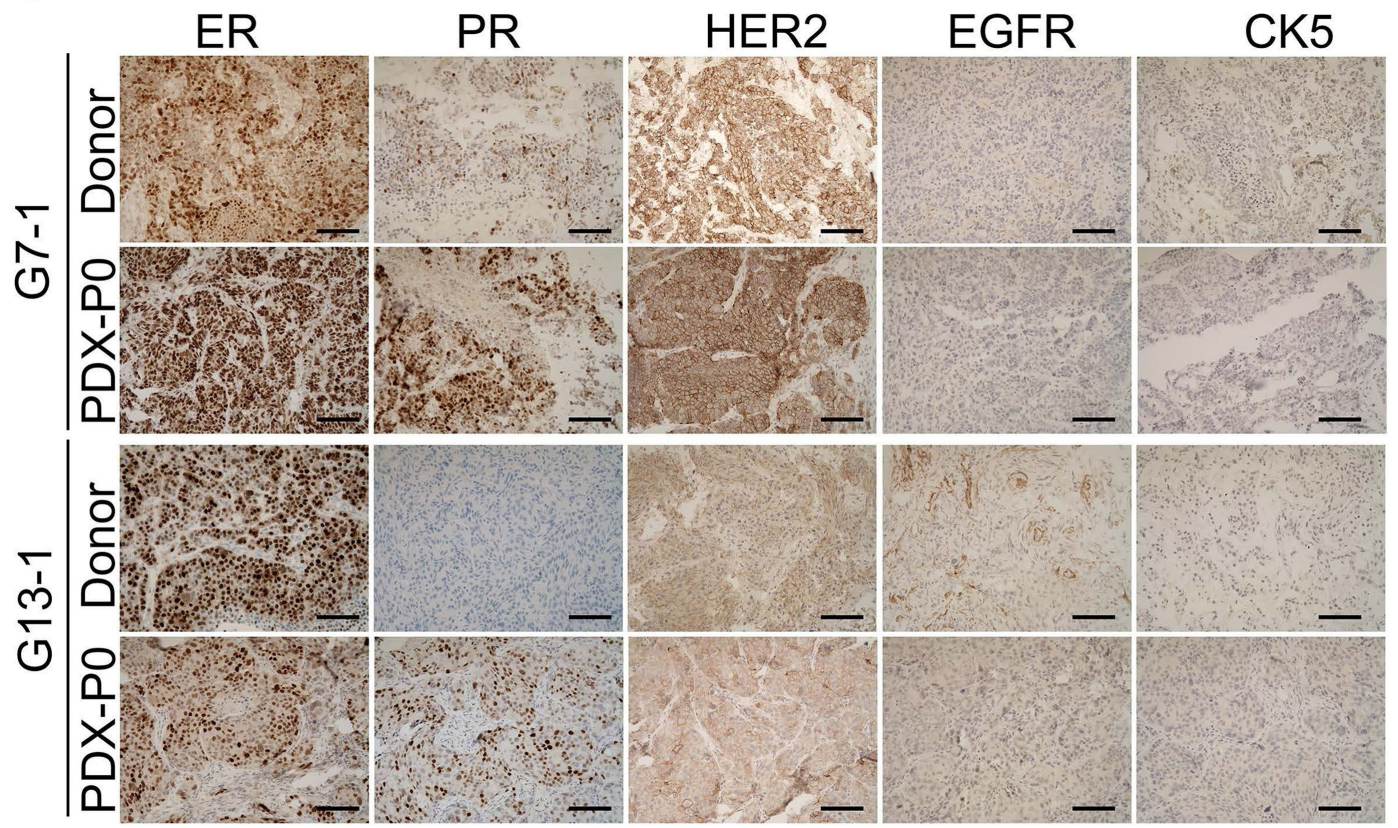

FIGURE 2 Retention of estrogen receptor (ER), progesterone receptor (PR), HER2, epidermal growth factor receptor (EGFR), and cytokeratin (CK) expression in brain-metastases-patient-derived xenografts (BM-PDXs). Sections of BM-PDXs were stained by IHC for ER, PR, HER2, EGFR, and CK5 at first passage (P0) and compared to donor tumor (when available). (A) Expression of EGFR, HER2, and CK5 in triple negative (TN) BM-PDXs (ER, PR, negative, not shown). (B) Expression of HER2, EGFR, and CK5 in HER2+BM-PDXs in donor and BM-PDXs PO. (C) Expression of ER, PR, HER2, EGFR, and CK5 in ER+HER2+BM-PDXs and their donor counterparts. Scale bars, $100 \mu \mathrm{m}$. 
A

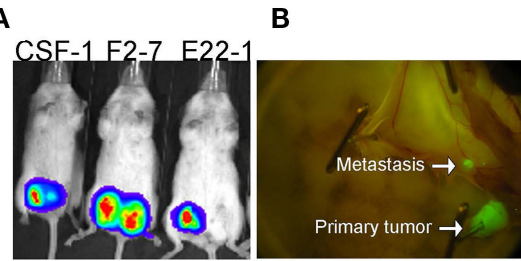

C
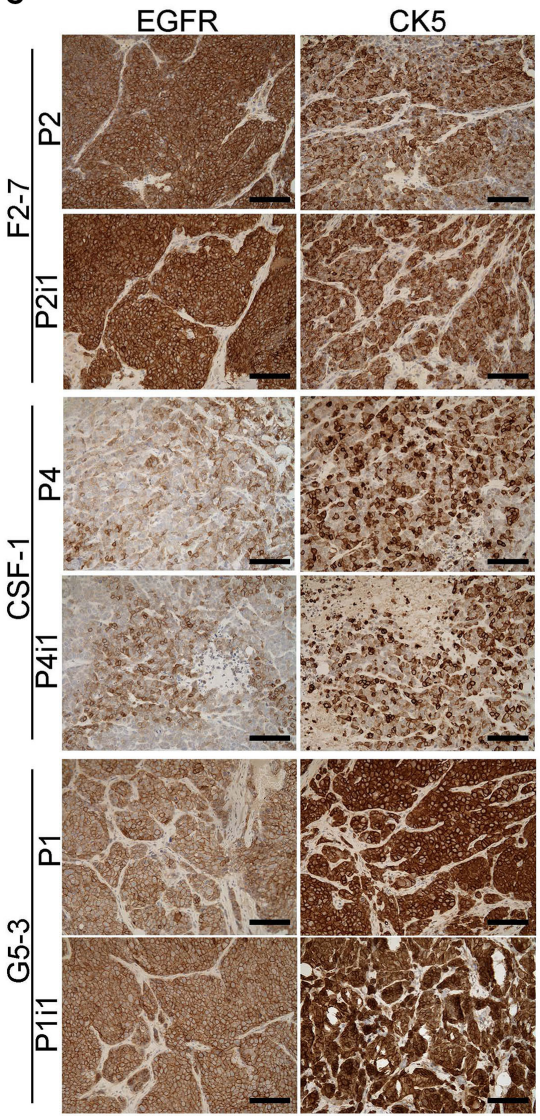

FIGURE 3 | GFP-Luciferase-labeled brain-metastases-patient-derived xenografts (BM-PDXs) retain expression of epidermal growth factor receptor (EGFR), cytokeratin 5 (CK5), and HER2. (A) Luciferase imaging (IVIS) of NSG mice carrying GFP-luciferase BM-PDXs F2-7, CSF-1, and G5-3 in the mammary fat pad. (B) Spontaneous metastases to lymph node in G5-3 GFPluc carrying mice. (C) Expression of EGFR and CK5 in sections of F2-7,

CSF-1, and G5-3 BM-PDXs before (P1, P2) and after GFP-luciferase labeling (P2-I1, P1-I1). Scale bars, $100 \mu \mathrm{m}$.

until mice showed $>15 \%$ weight loss or neurological impairment. Nine weeks after ic injection mice were imaged using T1/T2 MRI and mice without symptomatic metastases were left alive for 3 additional weeks. MRI-detectable brain metastases were found in $4 / 8(50 \%)$ of injected mice, with metastatic lesions ranking from 0.29 to $0.62 \mathrm{~mm}$ in size (Figure 6A, top). One additional mouse was injected with E22-BM-PDX dissociated cells but MRI was performed 14 weeks after ic injection. This mouse showed multiple large MRI-detectable metastases (Figure 6A, bottom). Histological analysis showed 8/8 mice (100\%) harboring micrometastases (defined as $>50 \mu \mathrm{m}$ cancer cell foci counted in six

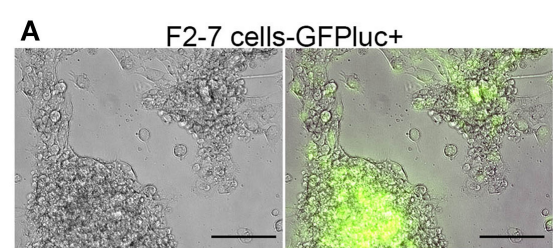

B

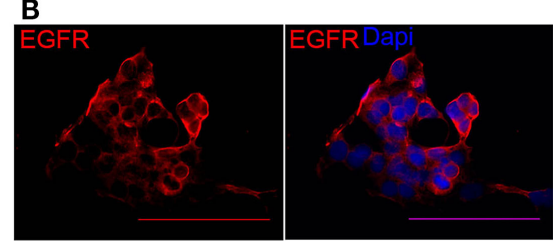

FIGURE 4 | Characteristics of F2-7 BM-cell line in vitro. (A) F2-7 cells were labeled in vitro with GFP-luciferase and cultured in collagen-I coated plates. Left: Brightfield, right: GFP expression of live cells. (B) Immunofluorescence staining shows epidermal growth factor receptor (EGFR) (red) expression in F2-7 cells grown in coverslips. 4',6-diamidino-2-phenylindole (DAPI) labels nuclei. Scale bars are $100 \mu \mathrm{m}$

sagittal brain sections $300 \mu \mathrm{m}$ apart) with a 10.25 median number of micrometastasis per mouse (Figure 6B). To determine whether brain metastases formed by BM-PDXs showed pathophysiological features similar to those encountered in humans, we performed double immunofluorescence staining of brain metastatic cells (pan-cytokeratin ${ }^{+}$, green) and reactive astrocytes $\left(\mathrm{GFAP}^{+}\right.$, red) or blood vessels (Col-IV, red) in brain sections from mice injected with E22-1 BM-PDXs. Brain metastatic clusters were located to the brain parenchyma (Figure 6C), were associated with blood vessels (Figure 6D) and were surrounded by $\mathrm{GFAP}^{+}$reactive astrocytes (Figure 6E); all of these characteristics of breast cancer brain metastases. Taken together, these studies demonstrate that BM-PDXs retain their ability to form large brain metastases and micro metastases at high frequencies, making them suitable models for studies of brain metastatic colonization and preclinical testing of drugs in preventive and therapeutic settings.

\section{DISCUSSION}

The increased incidence of brain metastasis in breast cancer patients and its dismal prognosis, has prompted the urgency to better understand the pathophysiology of brain metastases and to test novel therapeutic strategies for these patients. PDXs have emerged as required tools to validate in vitro studies in cells lines and to decipher the role of tumor heterogeneity in tumor progression and response to treatments (33-35). Therefore, we addressed whether PDX derived from brain-metastatic breast cancer are suitable models to study the pathophysiology of brain metastasis and to provide clinically relevant platforms for therapeutic drug testing. A diagram showing the overall procedure to achieve this from tumor implantation to ic injection of labeled cells is presented in Figure 7. By implanting fresh tumor samples in the mammary fat pad of NSG mice, we developed BM-PDXs from $\mathrm{TN}, \mathrm{ER}^{-} \mathrm{HER} 2^{+}, \mathrm{ER}^{+} \mathrm{HER} 2^{+}$, and $\mathrm{ER}^{+} \mathrm{HER} 2^{-}$subtypes. Consistent with the diverse incidence of brain metastasis among breast cancers subtypes (36-38), most specimens collected for 



FIGURE 5 | RNA expression profiles of F2-7 cell line and brain-metastases-patient-derived xenografts (BM-PDXs) before and after labeling. RNA sequencing of BM-PDX E22-1 prior to (E22-1-PDX-P0) and after GFP-luciferase labeling (E22-1 PDX-P0-11), BM-PDX CSF-1 P3, and F2-7 cell-line compared to F2-7 BM-PDX-P5. (A) Unsupervised hierarchical clustering of samples using the 500 most variable genes across all samples. (B) Normalized expression (log 2) plots of clinically relevant genes (ESR1, EGFR, PGR, ERBB2, NTRK2, KRT5). Differential expression was calculated using cufflinks (cuffdiff), and hierarchical clustering was performed in R.

implantation originated from TN and $\mathrm{HER} 2^{+}$tumors, and 6 of 8 established BM-PDXs were from TN and ER-HER2 ${ }^{+}$subtypes. Similarly, BM-PDXs from $\mathrm{ER}^{+}$patients (who show the lowest incidence of BMs) (39) showed the slowest progression when implanted as xenografts (Figure 1B), despite the fact that mice were supplemented with estradiol. Of clinical importance, two specimens had prior history of TNBC but their brain metastasis were reclassified as HER2 ${ }^{+}$by either immunohistochemistry or FISH. This is in agreement with recent reports of ERBB3/HER2 amplifications and mutations in breast cancer brain metastasis that are absent in primary tumors (40). This also highlights how changes in cancer cells occurring within the brain microenvironment modify tumor progression and impact their therapeutic alternatives.

Our BM-PDXs share characteristics of PDX models derived from primary tumors and other cancers. For example, our engraftment rate of $57.3 \%$ was similar to rates reported for engraftment of brain metastases from lung cancer (41). We also observed that the in vivo tumorigenic potential of patient-derived cancer cells was correlated with worse clinical outcome of patients. This is consistent with the idea that more aggressive/proliferating tumors are more likely to engraft as PDXs $(24,35)$. Unfortunately, these data also suggest that the potential use of personalized BM-PDX to test drug responses and guide clinical treatment, will not be feasible given the extremely short survival of those brain metastatic patients whose tumors grew as BM-PDXs (Figure 1C).

Similar to other studies, once established as PDXs, tumor cells appear to gain the ability to grow in vivo $(22,35)$, as demonstrated by the shorter time for BM-PDXs to develop into palpable tumors
(Figures 1D,E). While no apparent gain or loss of critical cell makers were observed between donor and $\mathrm{TN}$ and $\mathrm{ER}^{-} \mathrm{HER}{ }^{+} \mathrm{BM}-$ PDXs (Figures 2 and 5), it is possible that the increased growth rate represents differences in the initial number of cancer cells that proliferated to give rise to a PDX, rather than the selection of a subset of rapidly proliferating tumor cells. While we observed a high proportion of cells expressing the basal marker CK5 (a marker associated with a stem-like phenotype in breast cancer) $(32,42,43)$, we did not observe enrichment of $\mathrm{CK}^{+}$after passaging or cell dissociation, which could be interpreted as a selection of a more aggressive tumor clone. However, only genetic tracing of clonal populations within the tumors would allows to answer this question definitively. Our RNA sequencing data showing conserved expression of critical genes after PDX-cell dissociation (Figure 5) suggests that BM-PDXs can be manipulated in vitro (i.e., using CRISPR-cas9). This opens the window to use PDXs in mechanistic studies previously limited to cell line models.

Despite being expanded in the mammary fat pad, our BM-PDXs remain capable of colonizing the brain at high frequencies, suggesting that passaging tumors in the mouse does not decrease their brain metastatic potential. While the incidence of MRI-detectable metastases and micrometastases after ic injection were only measured in a cohort of mice injected with the E22-1 BM-PDXs, ongoing experiments in our laboratory suggest that this finding can be extended to the F2-7 cell line and G3-5 BM-PDXs (not shown). Importantly, brain metastases from E22-1 BM-PDX elicit astroglia activation (marked by expression of $\mathrm{GFAP}^{+}$astrocytes) and brain metastatic outgrowth around vessels in the brain parenchyma. Therefore, experimental metastases 

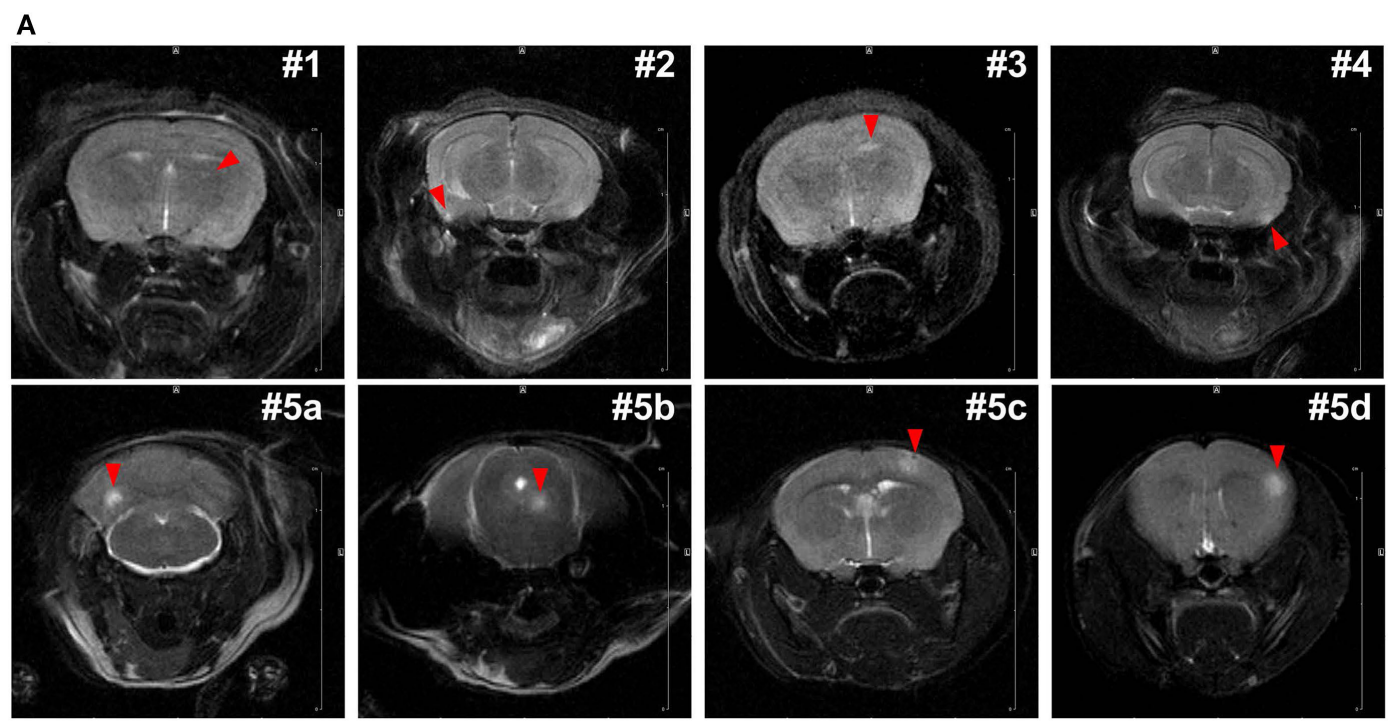

B

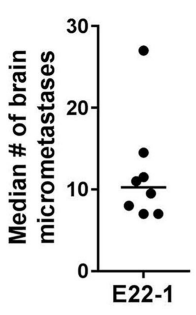

C



D

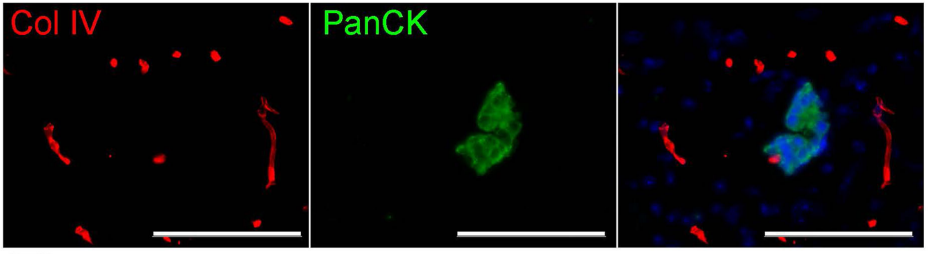

E
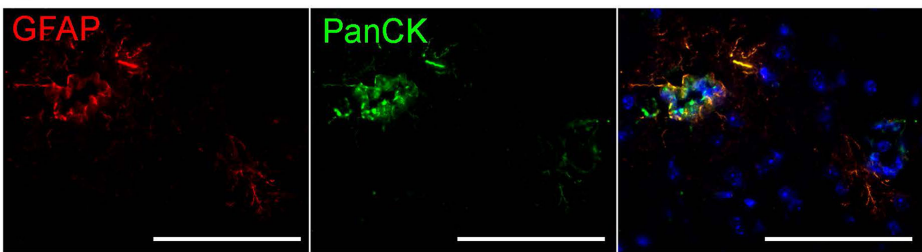

FIGURE 6 | Experimental metastases using brain-metastases-patient-derived xenografts (BM-PDXs). (A) Dissociated cells (250,000/100 $\mu$ l PBS) from BM-PDX E22-1 were injected intracardially in female NSG mice $(n=8)$. Representative T2-weigthed rapid acquisition with relaxation enhancement (RARE) magnetic resonance imaging (MRI) image shows large metastases in 4/10 mice, 8 weeks after injection (top panel). Lower panel shows large and multiple metastases brain metastases in one mouse at 13 weeks postinjection. The summed diameter of all metastases in this mouse was $3.7 \mathrm{~mm}$. (B) Median number of micrometastases per mouse, counted in six brain sections, $300 \mu \mathrm{m}$ apart. (C) H\&E staining shows micrometastasis in brain section from mice injected with E22-1 BM-PDX.

(D) Double immunofluorescence staining shows metastatic E22-1 cells (Pan-cytokeratin, PanCK, green) surrounded by reactive astrocytes (GFAP+, red) (20x).

(E) Double immunofluorescence staining shows PanCK+E22-1 cells outside of blood vessels (Col-IV) (20X).

with BM-PDXs recapitulate interactions with the brain microenvironment recognized as critical for brain metastatic success. Since we can genetically manipulate BM-PDXs dissociated cells or our F2-7 cell line, these novel models are now available to mechanistically assess how diverse breast tumors subtypes adapt to the brain microenvironment. More importantly, since PDXs show a slower progression rate than cell lines, these models are better suited for preclinical testing of drugs in a therapeutic setting, a task difficult to achieve in models where mice become moribund 3-4 weeks after injection.

It has been shown that breast cancer PDXs from primary tumors can colonize the brain if injected ic, suggesting that the intrinsic ability of tumor cells to colonize multiple organs is present in all PDXs regardless of site of origin. While our results indicate that $\mathrm{BM}-\mathrm{PDX}$ retain brain tropism, we observed spontaneous metastases of BM-PDXs from the orthotopic site to nearby vessels (Figure 4B) and in a few cases, metastases to bone and lungs after ic injection of BM-PDXs dissociated cells (not shown). This suggests, that similar to brain-homing cell lines and other PDXs, BM-PDXs maintain their ability to disseminate and colonize multiple metastatic sites (44). This also implies that ic injection of BM-PDXs might result in "undesired" metastases to other organs, which will limit our ability to measure brainmetastases-associated survival in these models. Recently, brainmetastatic PDX from lung cancer (41), melanoma (45) and HER2 ${ }^{+}$ breast cancer (46) were developed by direct intracranial injection of tumor samples in the brains of mouse or rats. Therefore, direct injection of dissociated cells from BM-PDXs might be an 


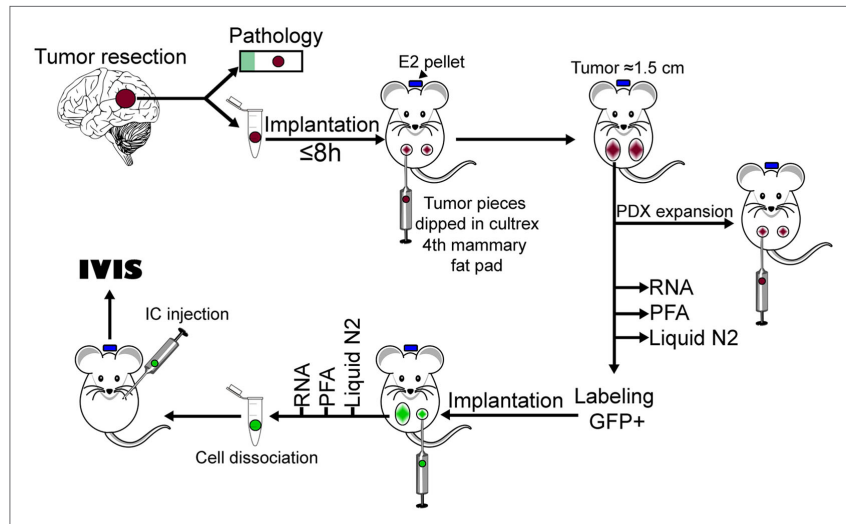

FIGURE 7 | Diagram of procedures used to develop brain-metastasespatient-derived xenografts (BM-PDXs) in this study.

alternative to induce a high frequency of brain metastases while minimizing the confounding effects of peripheral metastases in therapeutic studies. However, direct injection of cancer cells in the brain bypasses critical stages of brain metastastic colonization (hematogenous dissemination, intravasation, neuroinflammatory response, growth around vessels), which are hallmarks of breast cancer brain metastases. Intracarotid artery injection of cancer cells is a suitable alternative to ic injection for the production of brain-only metastasis-bearing mice with similar growth rates and mortality (47). Thus, we propose that intracarotid artery delivery of F2-7 cell line or BM-PDXs dissociated cells will enable the use of these heterogeneous models of brain metastatic breast cancer in mechanistic studies relevant to the pathophysiology of brain metastases, as well as to testing drug efficacy in preventive and therapeutic settings.

In conclusion, we developed and characterized eight novel PDX from breast cancer brain metastases from $\mathrm{ER}^{+}, \mathrm{HER}^{+}$, and TN subtypes, derived a matching cell line from one TN BM-PDX and demonstrated their brain metastatic potential. While all animal models harbor advantages and limitations, these novel BM-PDXs represent clinically relevant models that can be used to study how the heterogeneity of cancer cells affects brain colonization as well as for validation of therapies.

\section{ETHICS STATEMENT}

This study was carried out in accordance with the recommendations of the Department of Health and Human Services (HHS)

\section{REFERENCES}

1. Kaal ECA, Niël CGJH, Vecht CJ. Therapeutic management of brain metastasis. Lancet Neurol (2005) 4(5):289-98. doi:10.1016/S1474-4422(05)70072-7

2. Lin NU, Bellon JR, Winer EP. CNS metastases in breast cancer. JCO (2004) 22(17):3608-17. doi:10.1200/JCO.2004.01.175

3. Anders C, Carey LA. Understanding and treating triple-negative breast cancer. Oncology (Williston Park) (2008) 22(11):1233-9; discussion 9-40, 43.

4. Evans AJ, James JJ, Cornford EJ, Chan SY, Burrell HC, Pinder SE, et al. Brain metastases from breast cancer: identification of a high-risk group. Clin Oncol (R Coll Radiol) (2004) 16(5):345-9. doi:10.1016/j.clon.2004.03.012 regulations at 45 CFR 46 (also known as the "Common Rule") and the Food and Drug Administration (FDA) regulations at 21 CFR 50 and 21 CFR 56; as well as Department of Veterans Affairs policies for human research protection, including the regulations at 38 CFR 16, and the VHA Handbook 1200.05., with written consent from all subject. All subjects gave written informed consent in accordance with the Declaration of Helsinki. The protocol was approved by the Colorado Multiple Institutional Review Board (COMIRB), protocol \#13-3007 and the University of Colorado Denver Central Nervous System Biorepository Protocol, Steering Committee.

\section{AUTHOR CONTRIBUTIONS}

Conception and design: DC. acquisition of data (provided animals, acquired and managed patients, provided facilities, etc.): MC-Z, DO, AG, PK, CH, ND, KL, MG, VB, BJ, SE, AT, and DC. Analysis and interpretation of data (e.g., statistical analysis, biostatistics, imaging, computational analysis): MC-Z, AG, PK, NS, and DC. Administrative, technical, or material support: MC-Z, ND, CH, SE, DO, KL, MG, AT, PK, and DC. Study supervision: DC. All authors contributed to writing, review, and/or revision of the manuscript.

\section{ACKNOWLEDGMENTS}

The authors thank the Department of Neurosurgery Nervous System Biorepository personnel (Mary Wang, Jennifer Maitlen) for collection of fresh brain metastasis samples and administrative support, Kendra Huber at the UCCC animal imaging shared resources for MRI imaging, and the Tissue Biobanking and Histology Shared resources for their excellent services.

\section{FUNDING}

This work was supported by DOD BCRP W81XWH-11-1-0101 (DC), ACS IRG \# 57-001-53 (DC), and NCI K22CA181250 (DC). A grant from Drink for Pink and the Cancer League of Colorado supported MC-Z. The University of Colorado Cancer Center (UCCC) Animal Imaging Shared Resources, Tissue Culture Core, Tissue Biobanking and Histology Shares resource are supported by NCI P30CA046934 and CTSA UL1TR001082 Center grants. The University Of Colorado Department Of Neurosurgery funded the Nervous System Biorepository and sample collection.
5. Tham Y-L, Sexton K, Kramer R, Hilsenbeck S, Elledge R. Primary breast cancer phenotypes associated with propensity for central nervous system metastases. Cancer (2006) 107(4):696-704. doi:10.1002/cncr.22041

6. Hicks DG, Short SM, Prescott NL, Tarr SM, Coleman KA, Yoder BJ, et al. Breast cancers with brain metastases are more likely to be estrogen receptor negative, express the basal cytokeratin CK5/6, and overexpress HER2 or EGFR. Am J Surg Pathol (2006) 30(9):1097-104. doi:10.1097/01.pas.0000213306.05811.b9

7. Morris PG, Murphy CG, Mallam D, Accordino M, Patil S, Howard J, et al. Limited overall survival in patients with brain metastases from triple negative breast cancer. Breast J (2012) 18(4):345-50. doi:10.1111/j.1524-4741.2012. 01246.x 
8. Braccini AL, Azria D, Thezenas S, Romieu G, Ferrero JM, Jacot W. Prognostic factors of brain metastases from breast cancer: impact of targeted therapies. Breast (2013) 22(5):993-8. doi:10.1016/j.breast.2013.05.011

9. Tomasello G, Bedard PL, de Azambuja E, Lossignol D, Devriendt D, PiccartGebhart MJ. Brain metastases in HER2-positive breast cancer: the evolving role of lapatinib. Crit Rev Oncol Hematol (2010) 75(2):110-21. doi:10.1016/j. critrevonc.2009.11.003

10. Ghajar CM, Peinado H, Mori H, Matei IR, Evason KJ, Brazier H, et al. The perivascular niche regulates breast tumour dormancy. Nat Cell Biol (2013) 15(7):807-17. doi:10.1038/ncb2767

11. Eichler AF, Chung E, Kodack DP, Loeffler JS, Fukumura D, Jain RK. The biology of brain metastases-translation to new therapies. Nat Rev Clin Oncol (2011) 8(6):344-56. doi:10.1038/nrclinonc.2011.58

12. Hu G, Kang Y, Wang XF. From breast to the brain: unraveling the puzzle of metastasis organotropism. J Mol Cell Biol (2009) 1(1):3-5. doi:10.1093/jmcb/ mjp005

13. Marino N, Woditschka S, Reed LT, Nakayama J, Mayer M, Wetzel M, et al. Breast cancer metastasis: issues for the personalization of its prevention and treatment. Am J Pathol (2013) 183(4):1084-95. doi:10.1016/j.ajpath.2013.06.012

14. Palmieri D, Smith QR, Lockman PR, Bronder J, Gril B, Chambers AF, et al. Brain metastases of breast cancer. Breast Dis (2006) 26:139-47. doi:10.3233/ BD-2007-26112

15. Palmieri D, Bronder JL, Herring JM, Yoneda T, Weil RJ, Stark AM, et al. Her-2 overexpression increases the metastatic outgrowth of breast cancer cells in the brain. Cancer Res (2007) 67(9):4190-8. doi:10.1158/0008-5472.CAN06-3316

16. Munoz R, Man S, Shaked Y, Lee CR, Wong J, Francia G, et al. Highly efficacious nontoxic preclinical treatment for advanced metastatic breast cancer using combination oral UFT-cyclophosphamide metronomic chemotherapy. Cancer Res (2006) 66(7):3386-91. doi:10.1158/0008-5472.CAN-05-4411

17. Bos PD, Zhang XHF, Nadal C, Shu W, Gomis RR, Nguyen DX, et al. Genes that mediate breast cancer metastasis to the brain. Nature (2009) 459(7249):1005-9. doi:10.1038/nature08021

18. Zhang S, Huang WC, Zhang L, Zhang C, Lowery FJ, Ding Z, et al. SRC family kinases as novel therapeutic targets to treat breast cancer brain metastases. Cancer Res (2013) 73(18):5764-74. doi:10.1158/0008-5472.CAN-12-1803

19. Francia G, Cruz-Munoz W, Man S, Xu P, Kerbel RS. Mouse models of advanced spontaneous metastasis for experimental therapeutics. Nat Rev Cancer (2011) 11(2):135-41. doi:10.1038/nrc3001

20. Chen EI, Hewel J, Krueger JS, Tiraby C, Weber MR, Kralli A, et al. Adaptation of energy metabolism in breast cancer brain metastases. Cancer Res (2007) 67(4):1472-86. doi:10.1158/0008-5472.CAN-06-3137

21. Valastyan S, Weinberg RA. Tumor metastasis: molecular insights and evolving paradigms. Cell (2011) 147(2):275-92. doi:10.1016/j.cell.2011.09.024

22. Kabos P, Finlay-Schultz J, Li C, Kline E, Finlayson C, Wisell J, et al. Patientderived luminal breast cancer xenografts retain hormone receptor heterogeneity and help define unique estrogen-dependent gene signatures. Breast Cancer Res Treat (2012) 135(2):415-32. doi:10.1007/s10549-012-2164-8

23. DeRose YS, Wang G, Lin YC, Bernard PS, Buys SS, Ebbert MT, et al. Tumor grafts derived from women with breast cancer authentically reflect tumor pathology, growth, metastasis and disease outcomes. Nat Med (2011) 17(11):1514-20. doi:10.1038/nm.2454

24. Lum DH, Matsen C, Welm AL, Welm BE. Overview of human primary tumorgraft models: comparisons with traditional oncology preclinical models and the clinical relevance and utility of primary tumorgrafts in basic and translational oncology research. Curr Protoc Pharmacol (2012) 14:14.22. doi:10.1002/0471141755.ph1422s59

25. Tentler JJ, Tan AC, Weekes CD, Jimeno A, Leong S, Pitts TM, et al. Patientderived tumour xenografts as models for oncology drug development. Nat Rev Clin Oncol (2012) 9(6):338-50. doi:10.1038/nrclinonc.2012.61

26. Zhang H, Cohen AL, Krishnakumar S, Wapnir IL, Veeriah S, Deng G, et al. Patient-derived xenografts of triple-negative breast cancer reproduce molecular features of patient tumors and respond to mTOR inhibition. Breast Cancer Res (2014) 16(2):R36. doi:10.1186/bcr3640

27. Hanna C, Kwok L, Finlay-Schultz J, Sartorius CA, Cittelly DM. Labeling of breast cancer patient-derived xenografts with traceable reporters for tumor growth and metastasis studies. J Vis $\operatorname{Exp}$ (2016) 117:e54944. doi:10.3791/54944
28. Kim D, Pertea G, Trapnell C, Pimentel H, Kelley R, Salzberg SL. TopHat2: accurate alignment of transcriptomes in the presence of insertions, deletions and gene fusions. Genome Biol (2013) 14(4):R36. doi:10.1186/gb-2013-14-4-r36

29. Ahdesmaki MJ, Gray SR, Johnson JH, Lai Z. Disambiguate: an open-source application for disambiguating two species in next generation sequencing data from grafted samples. F1000Res (2016) 5:2741. doi:10.12688/ f1000research.10082.1

30. Liao Y, Smyth GK, Shi W. The subread aligner: fast, accurate and scalable read mapping by seed-and-vote. Nucleic Acids Res (2013) 41(10):e108. doi:10.1093/ nar/gkt214

31. Love MI, Huber W, Anders S. Moderated estimation of fold change and dispersion for RNA-seq data with DESeq2. Genome Biol (2014) 15(12):550. doi:10.1186/s13059-014-0550-8

32. Shao MM, Liu J, Vong JS, Niu Y, Germin B, Tang P, et al. A subset of breast cancer predisposes to brain metastasis. Med Mol Morphol (2011) 44(1):15-20. doi:10.1007/s00795-010-0495-2

33. Rosfjord E, Lucas J, Li G, Gerber HP. Advances in patient-derived tumor xenografts: from target identification to predicting clinical response rates in oncology. Biochem Pharmacol (2014) 91(2):135-43. doi:10.1016/j.bcp.2014.06.008

34. Siolas D, Hannon GJ. Patient-derived tumor xenografts: transforming clinical samples into mouse models. Cancer Res (2013) 73(17):5315-9. doi:10.1158/0008-5472.CAN-13-1069

35. Dobrolecki LE, Airhart SD, Alferez DG, Aparicio S, Behbod F, Bentires-Alj M, et al. Patient-derived xenograft (PDX) models in basic and translational breast cancer research. Cancer Metastasis Rev (2016) 35(4):547-73. doi:10.1007/ s10555-016-9653-x

36. Tseng LM, Hsu NC, Chen SC, Lu YS, Lin CH, Chang DY, et al. Distant metastasis in triple-negative breast cancer. Neoplasma (2013) 60(3):290-4. doi:10.4149/neo_2013_038

37. Sihto H, Lundin J, Lundin M, Lehtimaki T, Ristimaki A, Holli K, et al. Breast cancer biological subtypes and protein expression predict for the preferential distant metastasis sites: a nationwide cohort study. Breast Cancer Res (2011) 13(5):R87. doi:10.1186/bcr2944

38. Weil RJ, Palmieri DC, Bronder JL, Stark AM, Steeg PS. Breast cancer metastasis to the central nervous system. Am J Pathol (2005) 167(4):913-20. doi:10.1016/ S0002-9440(10)61180-7

39. Hung MH, Liu CY, Shiau CY, Hsu CY, Tsai YF, Wang YL, et al. Effect of age and biological subtype on the risk and timing of brain metastasis in breast cancer patients. PLoS One (2014) 9(2):e89389. doi:10.1371/journal.pone.0089389

40. Priedigkeit N, Hartmaier RJ, Chen Y, Vareslija D, Basudan A, Watters RJ, et al. Intrinsic subtype switching and acquired ERBB2/HER2 amplifications and mutations in breast cancer brain metastases. JAMA Oncol (2017) 3(5):666-71. doi:10.1001/jamaoncol.2016.5630

41. Lee HW, Lee JI, Lee SJ, Cho HJ, Song HJ, Jeong DE, et al. Patient-derived xenografts from non-small cell lung cancer brain metastases are valuable translational platforms for the development of personalized targeted therapy. Clin Cancer Res (2015) 21(5):1172-82. doi:10.1158/1078-0432.CCR-14-1589

42. Sartorius CA, Harvell DM, Shen T, Horwitz KB. Progestins initiate a luminal to myoepithelial switch in estrogen-dependent human breast tumors without altering growth. Cancer Res (2005) 65(21):9779-88. doi:10.1158/0008-5472. CAN-05-0505

43. Cittelly DM, Finlay-Schultz J, Howe EN, Spoelstra NS, Axlund SD, Hendricks P, et al. Progestin suppression of miR-29 potentiates dedifferentiation of breast cancer cells via KLF4. Oncogene (2013) 32(20):2555-64. doi:10.1038/onc.2012.275

44. Giuliano M, Herrera S, Christiny P, Shaw C, Creighton CJ, Mitchell T, et al. Circulating and disseminated tumor cells from breast cancer patient-derived xenograft-bearing mice as a novel model to study metastasis. Breast Cancer Res (2015) 17:3. doi:10.1186/s13058-014-0508-5

45. Wang J, Daphu I, Pedersen PH, Miletic H, Hovland R, Mork S, et al. A novel brain metastases model developed in immunodeficient rats closely mimics the growth of metastatic brain tumours in patients. Neuropathol Appl Neurobiol (2011) 37(2):189-205. doi:10.1111/j.1365-2990.2010.01119.x

46. Ni J, Ramkissoon SH, Xie S, Goel S, Stover DG, Guo H, et al. Combination inhibition of PI3K and mTORC1 yields durable remissions in mice bearing orthotopic patient-derived xenografts of HER2-positive breast cancer brain metastases. Nat Med (2016) 22(7):723-6. doi:10.1038/nm.4120 
47. Zhang C, Lowery FJ, Yu D. Intracarotid cancer cell injection to produce mouse models of brain metastasis. J Vis Exp (2017) 120:e55085. doi:10.3791/ 55085

Conflict of Interest Statement: The authors declare that the research was conducted in the absence of any commercial or financial relationships that could be construed as a potential conflict of interest.
Copyright $\odot 2017$ Contreras-Zárate, Ormond, Gillen, Hanna, Day, Serkova, Jacobsen, Edgerton, Thor, Borges, Lillehei, Graner, Kabos and Cittelly. This is an open-access article distributed under the terms of the Creative Commons Attribution License (CC BY). The use, distribution or reproduction in other forums is permitted, provided the original author(s) or licensor are credited and that the original publication in this journal is cited, in accordance with accepted academic practice. No use, distribution or reproduction is permitted which does not comply with these terms. 